\title{
PARAMETRIC MODELING AND FAULT TOLERANT CONTROL ${ }^{\dagger}$
}

\author{
N. Eva Wu and Jianhong Ju \\ Department of Electrical Engineering, Binghamton University, Binghamton, NY 13902-6000 \\ Telephone: (607) 777-4375, Fax: (607) 777-1931, Email: bg24615,evawu@binghamton.edu
}

\begin{abstract}
Fault tolerant control is considered for a nonlinear aircraft model expressed as a linear parametervarying system. By proper parameterization of foreseeable faults, the linear parameter-varying system can include fault effects as additional varying parameters. A recently developed technique in fault effect parameter estimation $^{[14]}$ allows us to assume that estimates of the fault effect parameters are available on-line. Reconfigurability ${ }^{[12]}$ is calculated for this model with respect to the loss of control effectiveness to assess the potentiality of the model to tolerate such losses prior to control design. The control design is carried out by applying a polytopic method ${ }^{[2,3]}$ to the aircraft model. An error bound on fault effect parameter estimation is provided, within which the Lyapunov stability of the closed-loop system is robust. Our simulation results show that as long as the fault parameter estimates are sufficiently accurate, the polytopic controller can provide satisfactory fault-tolerance.
\end{abstract}

\section{Introduction}

A linear parameter varying (LPV hereafter) system is a linear time-varying process whose state-space matrices are functions of some vector $p$ of timevarying parameters. An LPV system takes the form

$$
\begin{aligned}
& \dot{x}=A(p) x+B(p) u \\
& y=C(p) x+D(p) u
\end{aligned}
$$

where $p$ is assumed to change in a set in a parameter space. Several recently developed synthesis methods allow the design of global control laws for LPV systems ${ }^{[2,3,10,11]}$. Some of the self-scheduled methods furthermore provide theoretical guarantees in terms of both stability and performance in the presence of fast time domain evolution of the scheduled variables. Two such control design methods are the polytopic LVP method $^{[2,3]}$ and the linear fractional transformation (LFT) LPV method ${ }^{[10]}$. Comparison of these two methods is made in [9], where it is shown that each work has its merits and limits.

By properly parameterizing the faults, linear parameter-varying systems can include fault effects as additional varying parameters. These parameters will be called fault effect parameters in this paper.
Therefore it is possible to formulate a fault tolerant control design problem as an LPV control problem where the varying parameters representing faults are estimated on-line. The algorithm for fault effect parameter estimation given in [15] has been applied to est mate additive, multiplicative, and incipient faults simultaneously ${ }^{[16]}$. It is assumed in this paper that fault effect parameter estimates and their error bounds are available.

To distinguish an LPV system defined in the conventional sense, and one that include fault effect parameters, the abbreviation FLPV will be used in the paper to denote the latter, where $F$ indicates that some of the varying parameters are fault effect parameters. Unlike the case of an LPV system, where the varying parameters are measured in realtime, the only usable FLPV models are ones where the fault effect parameters are estimated quantities in real-time. When estimation errors of fault effect parameters become severe, properties that hold for LPV systems may no longer hold for FLPV. Therefore, an attempt is made in this paper to quant fy the allowable fault effect estimation errors in order to apply the LPV control design techniques to FLPV systems.

An FLPV system takes the form

\footnotetext{
${ }^{+}$This work was supported by grants from NASA(NCC-1-336), and NSF(ECS-91515956).
} 


$$
\begin{aligned}
& \dot{x}=A_{f}(p, \theta) x+B_{f}(p, \theta) u \\
& y=C_{f}(p, \theta) x+D_{f}(p, \theta) u
\end{aligned}
$$

where subscript $f$ appears whenever the occurrence of faults is likely. In comparison with an LPV system, an FLPV system description has an additional parameter vector $\theta$ containing fault effect parameters. Our goal is to achieve fault tolerant control through the application of the polytopic LPV control method, where $A, B, C$, and $D$ depends affinely on $p$ and on $\theta$. An LFT based fault tolerant control approach is given in [7]. Three problems are encountered in applying the LPV polytopic control design method. First, parametric modeling of faults becomes necessary, which is followed by the need to estimate the fault effect parameters, and then the analysis on the consequence of the estimation error of fault effect parameters.

Our approach to solving the above problems is shown through a design example using the NASADryden model ${ }^{[6]}$. Section 2 discusses three aspects of modeling for fault tolerant control. The first is aimed at establishing the dependence of $A, B, C$, and $D$ on parameter vector $p$, which is an explicit function of the flight conditions. The second is aimed at establishing the dependence of $A, B, C$, and $D$ on parameter vector $\theta$, which is an explicit function of the foreseeable faults. The third is aimed at determining the reconfigurability ${ }^{[13]}$ which is a measure of the system ability to allow performance/stability restoration at the presence of faults. Section 3 of the paper carries out the control design for the FLPV form of the NASA-Dryden model derived in Section 2 following the LPV polytopic method ${ }^{[2]}$. In the FLPV model used, however, parameter vector $\theta$ is replaced by its estimate $\hat{\theta}$ because only $\hat{\theta}$ is known. Section 4 analyzes the effect of such replacement, and provides estimation error bounds within which closed-loop system stability is preserved. Section 5 presents the simulation results of a coordinated turn controlled by an FLPV-controller under several levels of reduced stabilator control effectiveness. Conclusions are given section 6 .

\section{Problem Formulation}

There are five control inputs (aileron, symmetric stabilat or, differential stabilator, rudder, and thrust) and nineteen measured states in the NASA-Dryden nonlinear aircraft model ${ }^{[6]}$. For the purpose of demonstration, a lateral linear parameter-varying model is extracted with altitude ranging from 17900 feet to 22400 feet and Mach number at 0.62 . The LPV system takes the form of

$$
\begin{aligned}
& \dot{x}=A(p) x+B u \\
& y=C x+D u
\end{aligned}
$$

Here the states are side-slip angle $\beta$, roll rate $p$, yaw rate $r$, bank angle $\phi$, left aileron position $A_{l}$, left stabilator position $S_{l}$ and rudder position $R_{u d}$. The primary control surfaces consist of differential stabilator $\left(\delta_{D}\right)$, rudder $\left(\delta_{R}\right)$ and aileron $\left(\delta_{A}\right)$. The main dynamic change resulting from the altitude change is reflected in the entries $a_{2 l}, a_{25}, a_{26}$, and $a_{3}$ in matrix $A$.

$$
\begin{aligned}
& A(p)= \\
& {\left[\begin{array}{lllllll}
-0.18 & 0.07 & -1.0 & 0.05 & -0.004 & -0.03 & 0.03 \\
-28.44+p_{1}-1.95 & 1.21 & 0 & 19.52+2 p_{2} & 22.27+2 p_{3} & 1.49 \\
5.24+p_{4} & -0.08 & -0.53 & 0 & 0.26 & 1.31 & -2.97 \\
0 & 1 & 0.07 & 0 & 0 & 0 & 0 \\
0 & 0 & 0 & 0 & -20 & 0 & 0 \\
0 & 0 & 0 & 0 & 0 & -20 & 0 \\
0 & 0 & 0 & 0 & 0 & 0 & -20
\end{array}\right]} \\
& B=\left[\begin{array}{llll}
0 & 0 & 0 \\
0 & 0 & 0 \\
0 & 0 & 0 \\
0 & 0 & 10 \\
10 & 0 & 0 \\
0 & 20 & 0
\end{array}\right]
\end{aligned}
$$

In this case, one scheduling variable has generated four independent parameters in the $A(p)$ matrix, where $p_{1} \in[0,0.31], p_{2} \in[-2.9 .0], p_{3} \in[0,-3.2]$, and $p_{4} \in[-0.86,0]$. In general, one could determine an appropriate set where vector $p$ of an appropriate dimension resides in a parameter space corresponding to the range of the scheduling variables, such as altitude $h$ and Mach number $v$. The requirement of affine dependence generally 
results in a larger parameter set in a larger dimensional parameter space. Therefore, close attention should be paid to the common dependencies among the entries on the physical scheduling variables to reduce the number of independent parameters.

In case $B$ and/or $C$ also contain time-varying parameters, a pre-filter and post-filter can be added to form an augmented system where the augmented $A$ matrix absorbs all time-varying parameters.

\subsection{Parametric Modeling of Faults}

The example of loss of effectiveness in stabilator will be used for the discussion of parametric modeling of faults. Fault modeling turns an LPV process into an FLPV process. There are various possibilities that may result in control loss, which can lead to changes in $A$ matrix, or in $B$ matrix, or in both $A$ and $B$ matrices ${ }^{[11]}$. In our example, loss of stabilator control power causes a much more significant change in $B$ matrix than that in $A$ matrix. The FLPV model reflecting this condition is given by

$\dot{x}=A_{f}(p) x+B_{f}(\theta) u$

where $A(p)=A(p)$, and

$$
B_{f}(\theta)=\left[\begin{array}{llll}
0 & 0 & 0 & \\
0 & 0 & 0 & \\
0 & 0 & 0 & \\
0 & 0 & 0 & \\
0 & 0 & 10 & \\
10+100 & 0 & 0 \\
0 & 20 & 0 &
\end{array}\right]
$$

where $\theta$ is the fault effect parameter resulting from loss of stabilator effectiveness, which can be estimated in real-time ${ }^{[14]}$.

\subsection{Reconfigurability Computation}

In principle, both differential stabilators and ailerons can effectively control the aircraft roll movement. It is both interesting and enlightening to calculate the remaining control authority in the roll axis after a stabilator fault has occurred. The concept of reconfigurability measure and its compuation are given in [13]. The computation of reconfigurability amounts to solving for controllability gramian $W_{c}$ and observability gramian $w_{o}$ of the model for a specific value of $\theta$ value, obtaining the eigenvalues $\sigma_{1}^{2}, \sigma_{2}^{2}, \ldots, \sigma_{n}^{2}$ of $W_{c} W_{0}$, and determining the reconfigurability, defined as the smallest $\sigma_{i}(\theta)$ over a prescribed range of $\theta$. This is a measure of the ability of the proces; to be controlled to allow the restoration of performance with respect to the worst fault in a prescribed set of possible faults. The worst fault is defined in the sense that the largest effort in terms of energy is required in order to control the corresponding faulty system. Figure 3.1 shows the reconf igurability with respect to a stabilator control effectiveness loss of up to $80 \%$, while the aircraft altitude ranges from 17900 feet to 22400 feet.

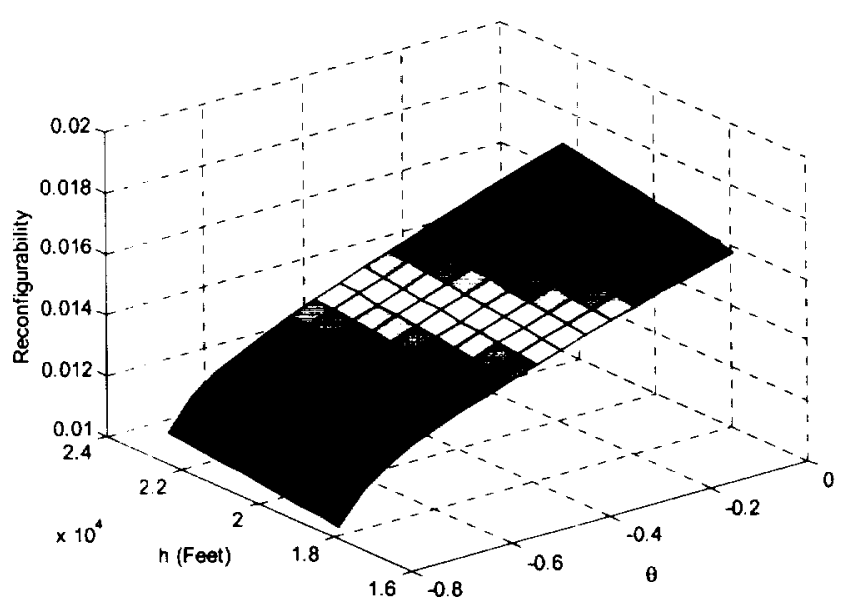

Figure 3.1 System reconfigurability versus altitude and control loss

It can be seen that the reconfigurability does not change much as the altitude varies, and it has over $65 \%$ control power remaining at an $80 \%$ loss of stabilator effectiveness. This reflects the fact that ailero 15 are there to back up the stabilators for the roll axis control. Study is ongoing with regard to obtairing a complete picture of reconfigurability with respect to various subsets of faults. 


\section{Controller Design}

In this section, a polytopic controller ${ }^{[2,3]}$ is designed for a FLPV description for the NASA-Dryden model where estimate $\hat{\theta}$ of parameter $\theta$ is assumed available in real time and replaces $\theta$ in the FLPV model.

A pre-filter of the form

$\dot{x}_{f i l}=A_{f i l} x_{f i l}+B_{f i l} \tilde{u}$

$u=C_{f i l} x_{f i l}$

and a post-filter of the form

$\dot{x}_{f i 2}=A_{f i 2} x_{f i 2}+B_{f i 2} y$

$\tilde{y}=C_{f i 2} x_{f i 2}$

can be used to remove the dependence of the inputto-state matrix $B$ and the state-to-output matrix $C$ on any time-varying parameters ${ }^{[4]}$. In the aircraft model under consideration, the augmented process model after introducing the pre-filter becomes

$$
\begin{aligned}
& {\left[\begin{array}{c}
\dot{x} \\
\dot{x}_{f i l}
\end{array}\right]=\left[\begin{array}{cc}
A_{f}(p) & B_{f}(\hat{\theta}) C_{f i l} \\
0 & A_{f i l}
\end{array}\right]\left[\begin{array}{c}
x \\
x_{f i l}
\end{array}\right]+\left[\begin{array}{c}
0 \\
B_{f i l}
\end{array}\right] \tilde{u} .} \\
& y=\left[\begin{array}{ll}
C & 0
\end{array}\right]\left[\begin{array}{c}
x \\
x_{f i l}
\end{array}\right]
\end{aligned}
$$

It can be seen that the only time-varying parameter dependent matrix is the stability derivative matrix. The filters must be designed so that its bandwidth is larger than the bandwidth of the desired closed-loop system and that the filters themselves must be stable. Once in the form of (3.1), the design of a self-scheduled controller can follow the standard procedure described in the LMI toolbox ${ }^{[8]}$. The controller has the following form

$$
\left(\begin{array}{ll}
A_{k}(p, \hat{\theta}) & B_{k}(p, \hat{\theta}) \\
C_{k}(p, \hat{\theta}) & D_{k}(p, \hat{\theta})
\end{array}\right)=\sum_{i=1}^{2^{n+r}} \alpha_{i}\left(\begin{array}{ll}
A_{k i} & B_{k i} \\
C_{k i} & D_{k i}
\end{array}\right)
$$

where $n$ is the dimension of parameter vector $p, r$ the dimension of parameter vector $\hat{\theta}, A_{k i}, B_{k i}, C_{k i}$, and $D_{k i}$ are the state-space parameters of an $H_{\infty}$ controller designed at $i^{\text {th }}$ vertex of the parameter polytope, subscript $k$ indicates that the parameter is associated with a controller, instead of the controlled process, $\alpha_{i}(t)$ 's are computed according to the sonvex decomposition of real time parameter values at each vertex, which meets the requirement $\left[\begin{array}{c}p(t) \\ \hat{\theta}(t)\end{array}\right]=\sum_{i=l}^{2^{n+r}} \alpha_{i}(t)\left[\begin{array}{c}p^{i}(t) \\ \hat{\theta}^{i}(t)\end{array}\right], \quad \sum_{i=1}^{2^{n+r}} \alpha_{i}(t)=1, \alpha_{i}>0$ where $p^{i}$ s and $\hat{\theta}^{i} \mathrm{~s}$ are the extreme values at $i^{\text {th }}$ vertex of the parameter ploytope, $n=4$ and $r=1$ in our example. Note that this setup depends on the availability of the estimate $\hat{\theta}$.

In [14], an adaptive filtering algorithm is developed for use to estimate the reduction of control effectiveness in a closed-loop setting. A set of covariance dependent forgetting factors is introduced into the filtering algorithm. As a result, the change in the control effectiveness is accentuated to help achieve a more accurate estimate more rapidly. A weighted sum-squared bias estimate is defined for the change detection. This method has been applied to fault diagnosis of a highly nonlinear ship propulsion system ${ }^{[15]}$, where addtive, multiplicative, as well as incipient faults are al parameterized, and the parameters are estimated. This paper assumes that both fault effect parameter estimates and their error bounds are proviced in real-time. The effect of time-delay of the usible estimates is not considered here.

\section{Roloustness against Estimation Error}

This section analyzes the effect of estimation error

$$
\tilde{\theta}=\theta-\hat{\theta}
$$

to the closed-loop system, in particular, the closedloop stability. To this end, parameter vector $p$ in the FLPV system matrices in (3.1) is suppressed. Suppose that the augmented process equations in the state-space form are

$$
\begin{aligned}
& \dot{x}=A^{\prime}(\theta) x+B u \\
& y=C x
\end{aligned}
$$

The affine dependence of $A$ on $\theta$ allows us to write

$$
A(\theta)=A(\theta=0)+\sum_{i=1}^{r} \theta_{i} A_{i}=A_{0}(\hat{\theta})+\Delta A(\tilde{\theta})
$$

where

$$
A_{0}(\hat{\theta})=A(\theta=0)+\sum_{i=I}^{r} \hat{\theta}_{i} A_{i}
$$

and 


$$
\Delta A(\tilde{\theta})=\sum_{i=1}^{r} \tilde{\theta}_{i} A_{i}
$$

The polytopic FLPV controller of appropriate order is described

$$
\begin{aligned}
& \dot{x}_{k}=A_{k}(\hat{\theta}) x_{k}+B_{k}(\hat{\theta}) y \\
& u=C_{k}(\hat{\theta}) x_{k}+D_{k}(\hat{\theta}) y
\end{aligned}
$$

The closed-loop system state equation in the absence of external input is

$$
\dot{x}_{c l}(t)=A_{c l}(\theta) x_{c l}(t)
$$

It is straightforward to show that

$$
\begin{aligned}
& A_{c l}=\left[\begin{array}{cc}
A(\theta) & 0 \\
0 & 0
\end{array}\right]+\left[\begin{array}{ll}
B & 0 \\
0 & I
\end{array}\right]\left[\begin{array}{cc}
D_{k}(\hat{\theta}) & C_{k}(\hat{\theta}) \\
B_{k}(\hat{\theta}) & A_{k}(\hat{\theta})
\end{array}\right]\left[\begin{array}{ll}
C & 0 \\
0 & I
\end{array}\right] \\
& =A_{c l}^{0}(\hat{\theta})+\Delta A_{c l}(\tilde{\theta}) \\
& \text { where } \\
& A_{c l}^{0}(\hat{\theta})=\left.A_{c l}\right|_{\theta=\hat{\theta}} \\
& =\left[\begin{array}{cc}
A_{0}(\hat{\theta}) & 0 \\
0 & 0
\end{array}\right]+\left[\begin{array}{ll}
B & 0 \\
0 & I
\end{array}\right]\left[\begin{array}{ll}
D_{k}(\hat{\theta}) & C_{k}(\hat{\theta}) \\
B_{k}(\hat{\theta}) & A_{k}(\hat{\theta})
\end{array}\right]\left[\begin{array}{ll}
C & 0 \\
0 & I
\end{array}\right]
\end{aligned}
$$

and

$$
\Delta A_{c l}(\tilde{\theta})=\left[\begin{array}{cc}
\Delta A(\tilde{\theta}) & 0 \\
0 & 0
\end{array}\right]
$$

Assume (4.2) is a uniform asymptotic stabilizing controller to (4.1) with $\theta$ replaced by $\hat{\theta}$, which results in the closed-loop state equation

$$
\dot{x}_{c l}(t)=A_{c l}(\hat{\theta}) x_{c l}(t)
$$

According to [1], for any continuous and bounded $Q(t)=Q(t)^{T}>0$, there exists

$$
P^{T}(t)=P(t)>0
$$

satisfying

$$
\dot{P}(t)=-Q(t)-A_{c l}^{0}(\hat{\theta})^{T} P(t)-P(t) A_{c l}^{0}(\hat{\theta})
$$

and

$$
V(t, x)=x_{c l}(t)^{T} P(t) x_{c l}(t)
$$

is a Lyapunov function with

$\dot{V}(t)=-x^{T}(t) Q(t) x(t)$

Theorem The closed-loop system in (4.3) is uniformly asymptotically stable if at any $t$

$$
\|\tilde{\theta}\|^{2}<\min \left(\sum_{i=l}^{r}\left\|A_{i}^{T} P_{I I}+P_{I I} A_{i}\right\|^{2}+2\left\|P_{I 2} A_{i}\right\|^{2}\right)^{-1}
$$

where
$\left[\begin{array}{ll}P_{11} & P_{12} \\ P_{12} & P_{22}\end{array}\right]=P$

is the solution of (4.5) at time $t$.

Proof. Assume that $A_{c l}^{0}(\hat{\theta})$ is uniformly asymptotically stable. Then (4.6) is a Lyapunov function for the system described by (4.4). Differentiating (4.6) with respect to $x_{c t}$ along the solution of (4.3) yields

$$
\begin{aligned}
& \dot{V}=\dot{x}_{c l}{ }^{T} P x_{c l}+x_{c l}{ }^{T} \dot{P} x_{c l}+x_{c l}{ }^{T} P \dot{x}_{c l} \\
& =x_{c l}{ }^{T}\left(\dot{P}+A_{c l}^{0}(\hat{\theta})^{T} P+P A_{c l}^{0}(\hat{\theta})\right) x_{c l} \\
& +x_{c l}{ }^{T}\left(\Delta A_{c l}(\tilde{\theta})^{T} P+P \Delta A_{c l}(\tilde{\theta})\right) x_{c l}
\end{aligned}
$$

Substituting (4.5) into the above equation gives

$$
\dot{V}=-\ddot{r}_{c l}^{T} Q x_{c l}+x_{c l}^{T}\left(\Delta A_{c l}(\tilde{\theta})^{T} P+P \Delta A_{c l}(\tilde{\theta})\right) x_{c l}
$$

For simplicity $Q=I$ is used. The requirement that $\dot{V}<0$ implies

$x_{c l}{ }^{T}\left(\Delta A_{c l}(\tilde{\theta})^{T} P+P \Delta A_{c l}(\tilde{\theta})\right) x_{c l}<x_{c l}{ }^{T} x_{c l}$

Since $\Delta A(\tilde{\theta})=\sum_{i=1}^{r} \tilde{\theta}_{i} A_{i}$ is affine in vector $\tilde{\theta}$,

imposes a convex constraint on $\tilde{\theta}$, and the set of all feasible solutions to the equation gives the bound on $\tilde{\theta}$.

Since

$$
\left|x_{c l}{ }^{T}\left(\Delta A_{c l}{ }^{T} P+P \Delta A_{c l}\right) x_{c l}\right| \leq x_{c l}{ }^{T} x_{c l}\left\|\Delta A_{c l}^{T} P+P \Delta A_{c l}\right\|
$$

and

$\left\|\Delta A_{c l}^{T} P+P A_{c l}\right\|^{2} \leq\left(\sum_{i=l}^{r}\left|\tilde{\theta}_{i}\right|\left\|\left[\begin{array}{cc}A_{i}^{T} P_{l I}+P A_{i} & A_{i}^{T} P_{12} \\ P_{12} A_{i} & 0\end{array}\right]\right\|^{2}\right.$

$\leq\|\tilde{\theta}\|^{2} \sum_{i=1}^{r}\left\|\left[\begin{array}{cc}A_{i}^{T} P_{l l}+P A_{i} & A_{i}^{T} P_{12} \\ P_{12} A_{i} & 0\end{array}\right]\right\|^{2}$

$\leq\|\tilde{\theta}\|^{2} \sum_{i=\Lambda}^{r}\left(\left\|A_{i}^{T} P_{11}+P A_{i}\right\|^{2}+\left\|P_{12} A_{i}\right\|^{2}\right)$

(4.7) holds if the right most term in the above inequality is less than 1 . Since $P$ is a function of time, a more conservative bound on the allowable estimation error is to force the maximum value of the right most term in the above inequality to be less than 1 . Therefore the claim of the theorem holds. 
In fact, if (4.4) satisfies the quadratic stability ${ }^{[5]}$, which is guaranteed by the existence of a constant $P=P^{T}>0$ such that (4.6) is a Lyapunov function, the calculation of the allowable estimation error bound can be simplified. Such constant $P$, if exists, can be obtained by applying a standard linear matrix inequality solver to the following problem

$$
P>0, \quad\left(A_{c l}^{j}\right)^{T} P+P A_{c l}^{j}<0, \quad j=1, \ldots, 2^{r}
$$

where $A_{c l}^{j} s$ describe the following polytopic linear differential inclusion

$\dot{x}_{c l}(t)=A_{c l}(\hat{\theta}) x_{c l}(t), \quad A_{c l}(\hat{\theta}) \in \operatorname{Co}\left\{A_{c l}^{l}, \cdots, A_{c l}^{2^{r}}\right\}$

In this case,

$\|\tilde{\theta}\|^{2}<\left(\sum_{i=1}^{r}\left\|A_{i}^{T} P_{11}+P_{11} A_{i}\right\|^{2}+2\left\|P_{12} A_{i}\right\|^{2}\right)^{-1}$

will guarantee the quadratic stability of the closedloop system. Note that the right hand side is now independent of time.

\section{Simulation Results}

Figure 6.1 shows the measured roll rates of a simulated scenario for the NASA-Dryden model a coordinated turn, carried out at an altitude of 18400 feet and an airspeed of $650 \mathrm{feet} / \mathrm{second}-$ using the stabilators and the rudder. The reference signal model is the same as that in [14] where much effort is devoted to the reference model selection. The stabilator is experiencing a control effectiveness loss at $0 \%, 15 \%$ and $50 \%$, respectively. The control effectiveness loss results in the stabilator position error directly. It is assumed that the control effectiveness loss estimate is accurate. From the roll rate response, it can be seen that the controlled system carries out the required task appropriately when the controller is scheduled according to the estimated fault effect parameter $\hat{\theta}$. It is also shown in Figure 6.1 that the required task cannot be fulfilled without treating the fault effect parameter as additional scheduled variables. When control effectiveness loss is at $15 \%$, and at $50 \%$, the polytopic FLPV control system without scheduling exhibits severely degraded performance, and becomes unstable, respectively. We therefore conclude that fault tolerance is achievable by using the polytopic FLPV controller.

Figure 6.2 shows the simulation results under a $15 \%$ loss of stabilator control effectiveness. This time different levels of control effectiveness estimation error are introduced. These level are $0 \%$, $50 \%, 100 \%$, and $150 \%$, respectively, with respect to the simulated control effectiveness loss. It is observed that the controlled system demonstrates a satisfactory performance when estimation error is sufficiently small, and the controlled system becomes unstable when the estimation error reaches. The actual allowable bound can be estimated using the formula provided in section 4 . Within the estimation error bound, the system performance degrades in terms of overshoot and tracking accuracy but the stability remains.

\section{Conclusions}

The polytopic FLPV method is used in this paper to achieve fault tolerant control demonstrated through the NASA-Dryden aircraft model ${ }^{[5]}$. The effects of faults in the aircraft, in particular, loss of stabilator control effectiveness, are modeled as affine timevarying parameters entering the parameters of the state space description. The system potentiality to allow the recovery of performance after a fault occurrence is investigated by carrying out the reconligurability computation. The polytopic contrcller is designed based on the FLPV model where the fault effect parameters take their estimated values. Bounds within which the closedloop system stability remains are derived for the fault effect parameter estimation errors. For the stabilator control loss case, it is observed that the polytopic FLPV controller offers satisfactory fault tolerance, provided that the estimation errors for fault effect parameters are sufficiently small. Our ongoing effort in this area includes further mode ing effort to obtain FLPV description of the aircraft model, development of robustness polytopic FLPV synthesis method with respect to fault effect parameter estimation, and the full scale nonlinear simulation of the controlled system. 


\section{References:}

1. B.D.O. Anderson, Exponential Stability of Linear Equations Arising in Adaptive Identifications, IEEE Transactions on Automatic Control, Vol.22, No.2, pp.83-88, 1977.

2. Pierre Apkarian, Pascal Gahinet \& Greg Becker, Self-scheduled $H_{\infty}$ Control of Linear Parameter-varying System: a Design Example, Automatica, vol.31, No.9, pp.1251-1261,1995.

3. Pierre Apkarian, Self-Scheduled $\mathrm{H}_{\infty}$ Control of Missile via Linear Matrix Inequality, Journal of Guidance, Control and Dynamics, Vol. 18, No. 3, pp.532-538, 1995.

4. G. Becker, A. Packard, D. Philbrick and G. Balas, Control of Parameter-dependent Linear Systems: a Single Quadratic Lyapunov Approach, American Control Conference, San Francisco, CA, pp. 2795-2799, 1993.

5. S. Boyd, L. El Ghaoui, E. Feron, and V. Balakrishnan, Linear Matrix Inequality in Systems and Control Theory, SIAM, 1994.

6. Randal W. Brumbaugh, Aircraft Model for the AIAA Controls Design Challenge, Journal of Guidance, Control and Dynamics, Vol. 17, No.4, pp. 747-752, 1994.

7. Jie Chen, R. J. Patton and Zheng Chen, Linear Matrix Inequality Formulation of Fault Tolerant Control System Design, $14^{\text {th }}$ Triennial World Congress of IFAC, Beijing, P.R.China, 1999.

8. Pascal Gahinet, Arkadi Nemirovski, Alan J. Laub and Mahmoud Chilali, LMI Control Toolbox, The Math Works, 1994.

9. Hiroyuki Kajiwara, LPV Techniques for Control of an Inverted Pendulum, IEEE control Systems Magazine, Vol.19, pp.44-54, 1999.

10. Andy Packard, Gain Scheduling via Linear Fractional Transformations, Systems and Control Letters, Vol. 22, , pp. 79-92,1994.

11. J. S. Shamma, Gain-Scheduling Missile Autopilot Design Using Linear Parameter Varying Transformation, Journal of Guidance,
Control and Dynamics, Vol. 16, No. 2, pp. 1317-1321, 1993.

12. Warren Weinstein, Control Reconfigurable Combat Aircraft Development Volum 3: Control Reconfigurable Handbook, Report to Wright Research and Development Center, \#AFWAL-TR-88-3118, 1990.

13. N. Eva Wu, Kemin Zhou and Gregory Salomon, On Reconfigurabilitty, to appear in Automatic, 2000.

14. N. Eva Wu, Vladimir Nikulin, Felix Heimes, and Victor Skormin, A Decentralized Adaptive Approach to Fault Tolerant Control, Proc. IF.4C Safeprocess, 2000.

15. N. Eva Wu, Youmin Zhang, and Kemin Zhou, Detection, Estimation and Accommodation of losis of Control Effectiveness, To appear in Iniernational Journal of Adaptive Control and Siz̧nal Processing, 2000.

16. Youmin Zhang, and $\mathrm{N}$. Eva Wu, Fault diagnosis for a ship propulsion benchmark: part I. Proc. IFAC 14th World Congress, 1999. 


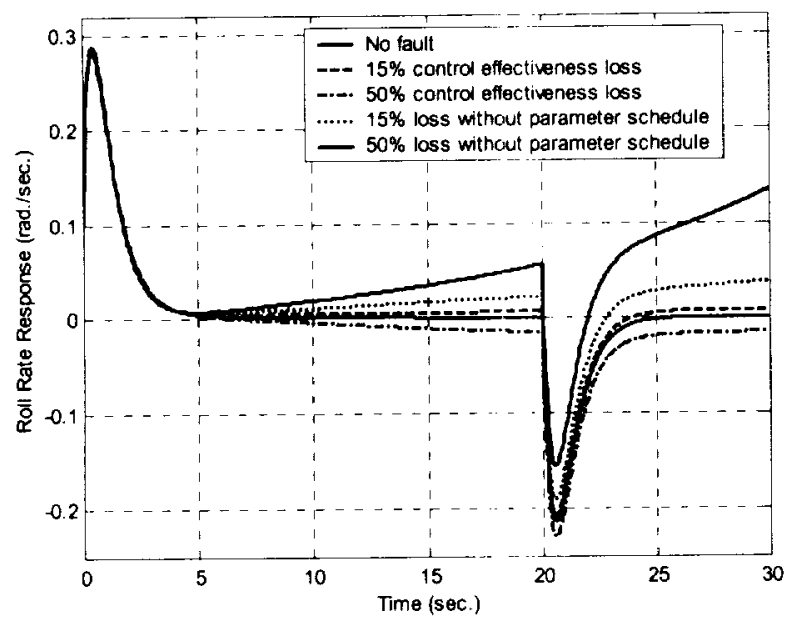

Figure 6.1 Roll rate response with stabilator control effectiveness loss accurately estimated

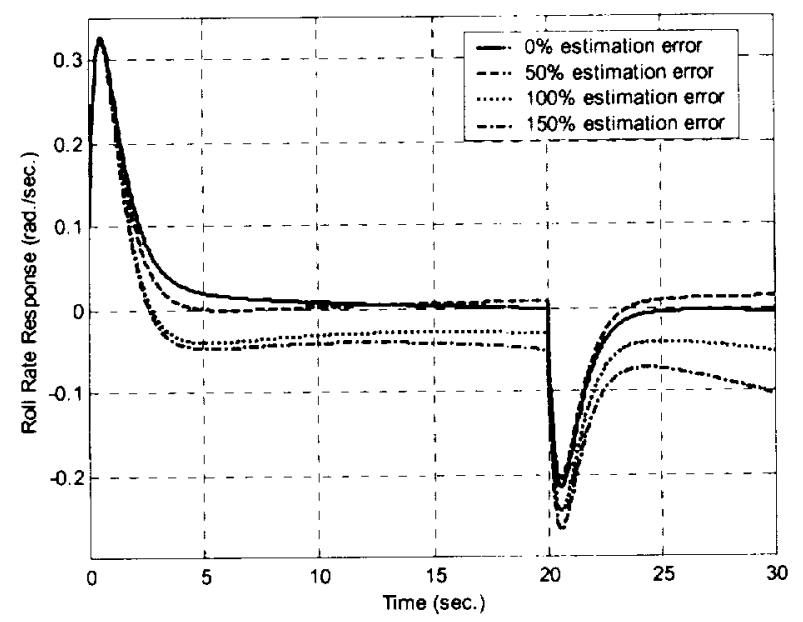

Fig 6.2 Roll rate response at $15 \%$ stabilator control effectiveness loss with estimation error 